\title{
COSPECTRAL GRAPHS AND DIGRAPHS
}

\author{
FRANK HARARY $\dagger$, CLARENCE KING, ABBE MOWSHOWITZ AND \\ RONALD C. READ
}

\section{Introduction}

At a meeting of the American Mathematical Society, one of us hazarded a conjecture that the characteristic polynomial of the adjacency matrix of a graph characterizes the graph, that is, that two graphs are isomorphic if and only if the characteristic polynomials of their adjacency matrices are the same. The conjecture was promptly shot down by a member of the audience (R. C. Bose), who produced a counter-example. In fact, it was already a matter of public record that the conjecture was not true, since Collatz and Sinogowitz [1] had displayed two different trees with 8 nodes having the same characteristic polynomial. In what follows we give an account of some recent computer investigations of the smallest graphs and digraphs of various kinds whose adjacency matrices have the same characteristic polynomial, in order to illuminate the extent to which the characteristic polynomial can be used to characterize a graph.

\section{Preliminaries}

Graph theoretic definitions not included here may be found in [2]. The adjacency matrix of a digraph $D$ with nodes $v_{1}, v_{2}, \ldots, v_{p}$ is the matrix $A=A(D)=\left(a_{i j}\right)$ where

$$
a_{i j}=\left\{\begin{array}{l}
1 \text { if there is an arc from } v_{i} \text { to } v_{j} \\
0 \text { otherwise. }
\end{array}\right.
$$

Noting that a graph may be regarded as a symmetric digraph, this also serves to define the adjacency matrix of a graph.

For brevity, the characteristic polynomial of the adjacency matrix of a graph or digraph will be called the characteristic polynomial of the graph or digraph. If two graphs or digraphs have the same characteristic polynomial we will say that they are cospectral.

\section{Digraphs}

Concepts about digraphs used here are defined in the book [3]. Since the characteristic polynomial of a block upper triangular matrix depends only on the elements in the diagonal blocks, many different cospectral digraphs can be constructed merely by filling in the above-diagonal blocks in an arbitrary manner. Since the characteristic polynomials of any digraph is the product of the characteristic polynomials of its strong components, we turn to the problem of finding a distinct pair of strongly connected digraphs. It is not difficult to verify by exhaustion that there are no such pairs of digraphs with less than 4 nodes, and at this stage we can

Received 19 April, 1971.

$\dagger$ Research supported in part by a grant from the Air Force Office of Scientific Research.

[Bull. LoNDon MATH. Soc., 3 (1971), 321-328] 
turn to a list of digraphs with 4 nodes recently prepared on an IBM 1620 computer (see [6]). A perusal of this list, which includes the connectedness categories, turns up the two digraphs shown in Fig. 1, which are both strong and have the same characteristic polynomial, $\lambda^{4}-\lambda^{2}-\lambda-1$, for if we reverse all the arrows on a digraph, we obtain in general a different digraph-the converse of the original. Clearly, a digraph and its converse are cospectral since their adjacency matrices are transposes of each other. The digraphs in Fig. 1 provide an example of such a pair; these are two smallest strong digraphs which are not self-converse.
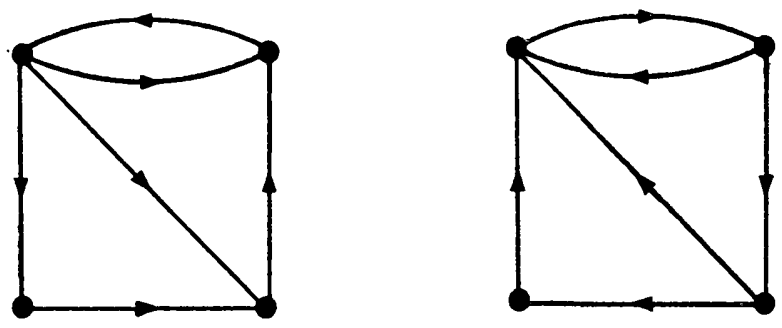

Fig. 1

Hence it is of interest to look for an example of two strong digraphs, not converse of each other, which are cospectral. Consulting the list again we find the strong digraph of Fig. 2, which has the same characteristic polynomial as the two digraphs in Fig. 1, but is not a converse of either of them. We have, incidentally, exhibited a triad of cospectral strong digraphs; on including the converse of Fig. 2, a tetrad.

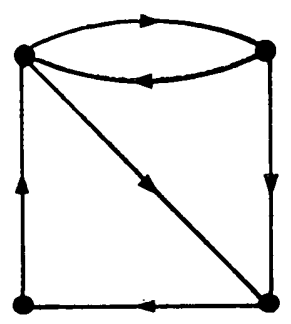

Fig. 2

\section{Graphs}

The adjacency matrix of a graph is symmetric and hence cannot be block upper triangular. There is no known short cut in the search for cospectral graphs.

Accordingly, the IBM 1620 computer at the University of the West Indies was programmed to produce a list of the characteristic polynomials of all graphs on 7 nodes, using as input the cards for these graphs prepared by B. R. Heap of the National Physical Laboratory. This list contains all graphs, connected or not, including those with isolated nodes. Hence the resulting polynomials yield all the cospectral pairs of graphs on 7 nodes, or less. The production of this list is described in [4]. 
Several questions can then be answered concerning the smallest pairs of cospectral graphs of a given kind. The minimum number of nodes for which cospectral graphs exist is 5; these are shown as the two graphs in Fig. 3, which both have the characteristic polynomial $\lambda^{5}-4 \lambda^{3}$. This pair of giaphs was also discovered by D. Rosenfeld of the Hebrew University and others.
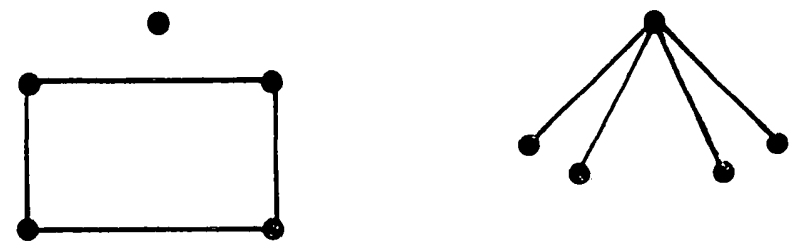

Fig. 3

One of these two cospectral graphs is not connected, and it is natural to look for the smallest pair of cospectral connected graphs. This is provided by the two connected graphs on 6 nodes shown in Fig. 4, both having characteristic polynomial $\lambda^{6}-7 \lambda^{4}-4 \lambda^{3}+7 \lambda^{2}+4 \lambda-1$.

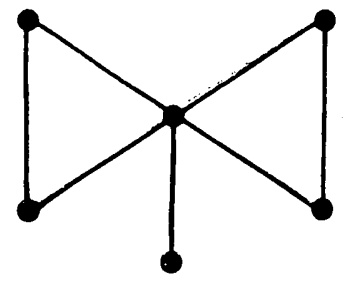

We can also ask for the smallest example of three cospectral connected graphs. This is given by the seven-point graphs in Fig. 5, with characteristic polynomial $\lambda^{7}-11 \lambda^{5}-10 \lambda^{4}+16 \lambda^{3}+16 \lambda^{2}$.

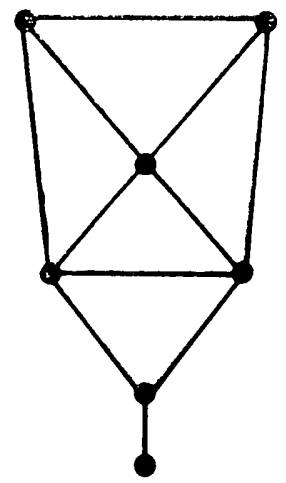

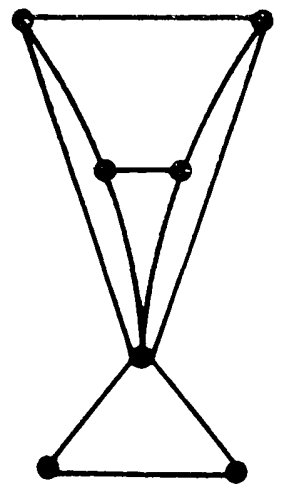

Fig. 5 
No tetrads occur among the 7-node graphs. However, their existence among $p$-node graphs for some $p>7$ is assured as a consequence of the following (see [5]).

THEOREM 1. For any positive integer $n$, there exists an integer $p$ such that there are $n$ cospectral graphs with $p$ nodes.

Another natural question is to ask for the smallest example of two cospectral trees. As mentioned above, the answer is given in Collatz and Sinogowitz [1]; (see Fig. 6). It is worth noting in this connection that the list of the coefficients of the characteristic polynomial of trees with $p \leqslant 8$ nodes as given in [1] contains two errors. A revised and expanded list $(p \leqslant 10)$ appears in [5].
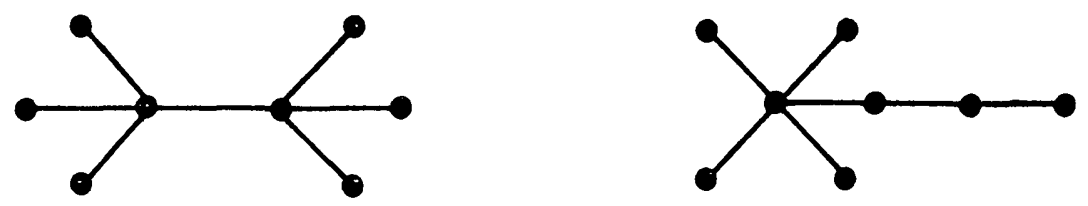

Fig. 6

In all the above examples (Figs. 3-6) of cospectral graphs, it is clear that the graphs are non-isomorphic since their partitions (the degree sequences of the nodes) are different. There is no pair of cospectral 7-node graphs with the same partition, but such pairs can occur in bigger graphs, as demonstrated in Fig. 7 with two cospectral trees. It is not known whether this example of a pair of cospectral graphs with the same partition gives the smallest possible such pair, although it is a smallest pair of cospectral trees having this property.
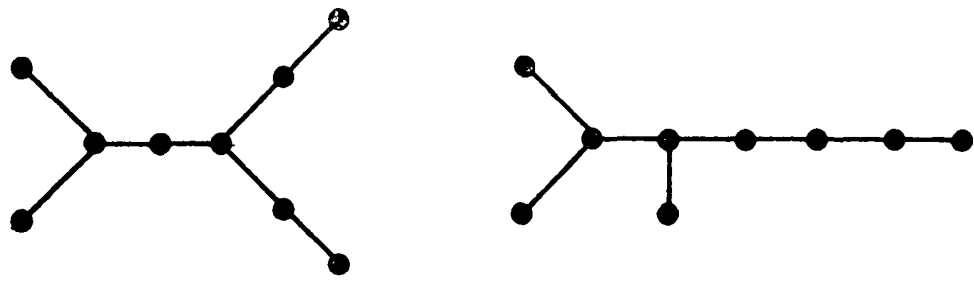

Fig. 7

\section{Some specific characteristic polynomials}

It will be of interest to list here the characteristic polynomials of some common types of graphs. We write the characteristic polynomial of a graph $G$ as $\phi(G)$, the variable $\lambda$ being understood. The following characteristic polynomials for the complete graph $K_{n}$, the $n$-cycle $C_{n}$, the $n$-cube $Q_{n}$, and the complete $t$-partite graph $K\left(n_{1}, n_{2}, \ldots, n_{t}\right)$ were calculated by M. D. Plummer and are readily verified:

$$
\begin{aligned}
& \phi\left(K_{n}\right)=(\lambda+1)^{n-1}(\lambda-n+1), \\
& \phi\left(C_{n}\right)=\prod_{k=1}^{n}\left\{\lambda-2\left(\cos \frac{2 k \pi}{n}\right)\right\},
\end{aligned}
$$




$$
\begin{gathered}
\phi\left(Q_{n}\right)=\prod_{k=0}^{n}\{\lambda-(n-2 k)\}^{(n / k)} \\
\phi\left(K\left(n_{1}, n_{2}, \ldots, n_{t}\right)\right)=\sum_{r=0}^{t}(1-r) S_{r}\left(n_{1}, n_{2}, \ldots, n_{t}\right) \lambda^{n-t},
\end{gathered}
$$

where $S_{r}\left(n_{1}, n_{2}, \ldots, n_{t}\right)$ denotes $\dagger$ the sum of the products $r$ at a time of the numbers $n_{1}, n_{2}, \ldots, n_{t}$, and $S_{0}=1$.

As corollaries of (4) we have the characteristic polynomial for the complete bipartite graph $K_{m, n}$, viz.

and the $\operatorname{star} K_{1, n}$, viz.

$$
\Phi\left(K_{m, n}\right)=\lambda^{m+n-2}\left(\lambda^{2}-m n\right)
$$

$$
\Phi\left(K_{1, n}\right)=\lambda^{n-1}\left(\lambda^{2}-n\right) .
$$

The calculation of characteristic polynomials for graphs of any size is usually extremely tedious, but there is a short cut which can be applied to any graphs having a node of degree 1 , and in particular to trees. This depends on the following theorem, in which $G-u$ is the graph obtained from $G$ by deleting the end node $u$, and $G-u v$ denotes the graph $G$ without the edge $u v$.

Clearly, since $G-u v$ has $u$ as an isolated node, we have $\phi(G-u v)=\lambda \phi(G-u)$, and we may write $\phi(G)=\lambda \phi(G-u)-\lambda(G-u-v)$.

THEOREM 2. If $u$ is a node of degree 1 in a graph $G$, and $v$ is the node of $G$ to which it is adjacent, then

$$
\phi(G)=\phi(G-u v)-\phi(G-u-v) .
$$

Proof. Without loss of generality we can take $u$ and $v$ as nodes 1 and 2 in setting up the adjacency matrix.

Expanding by the first row we have

$$
\begin{aligned}
& \phi(G)=\lambda\left[\begin{array}{cccc}
\lambda & -a_{23} & -a_{24} & \cdots \\
-a_{23} & \lambda & \cdot & \cdots \\
-a_{24} & . & . & \cdots
\end{array}\right]+\left[\begin{array}{cccc}
-1 & -a_{23} & -a_{24} & \cdots \\
0 & \lambda & -a_{34} & \cdots \\
0 & -a_{34} & \lambda & \cdots \\
& \cdots & &
\end{array}\right] \\
& =\lambda \phi(G-u)-\left[\begin{array}{ccc}
\lambda & -a_{34} & \cdots \\
-a_{34} & \lambda & \cdots \\
\cdot & \cdot & \cdots
\end{array}\right] \\
& =\phi(G-u v)-\phi(G-u-v) \text {. }
\end{aligned}
$$

In applying this theorem we can profitably make use of a notational device introduced by Zykov [7] for chromatic polynomials, and allow the drawing of a

$\dagger S_{r}$ is sometimes called the $r$ th symmetric function. 
graph to stand for its characteristic polynomial. Thus if we wished to apply the theorem to the graph of Fig. 8, we could set out the computation as in Fig. 9

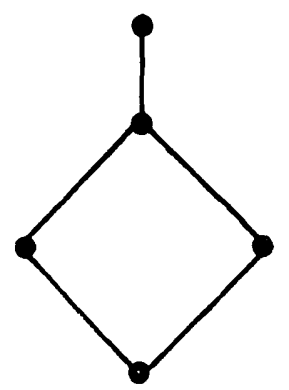

Fig. 8

The characteristic polynomial is now expressed as $\lambda \phi\left(G_{4}\right)-\phi\left(K_{1},{ }_{2}\right)$, that is, in terms of known polynomials by (2) and (6), so that it is easily computed to be $\lambda^{5}-2 \lambda^{3}+2 \lambda$.

If the graphs on the right-hand side of an equation like (7) have end nodes, the theorem can be applied again, but this will not be possible in general. For trees, however, this is always possible, and the computation can be carried out in full. A further result can be deduced very readily from Theorem 2 , namely the characteristic polynomial of the $n$-path $P_{n}$, the tree on $n$ nodes with partition $1^{2} 2^{n-2}$.
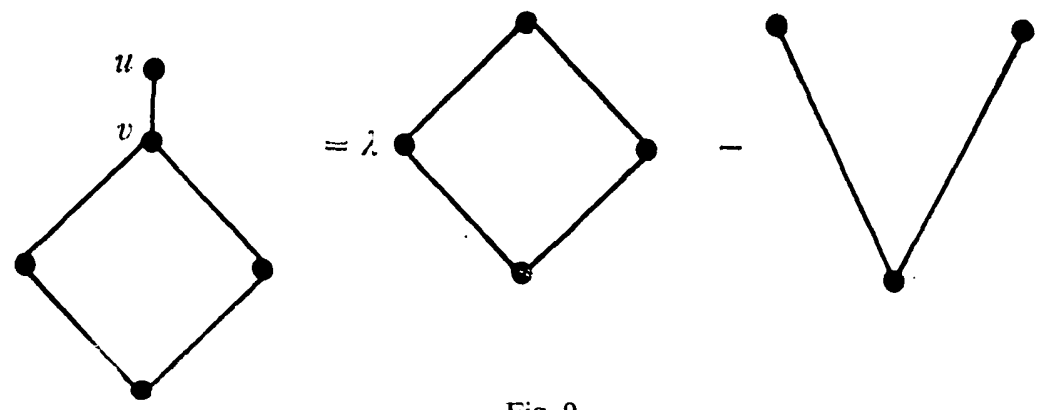

Fig. 9

THEOREM 3. The characteristic polynomial of $P_{n}$ satisfies the recurrence relation

$$
\phi\left(P_{n}\right)-\lambda \phi\left(P_{n-1}\right)-\phi\left(P_{n-2}\right) \text {. }
$$

Proof. This is a direct consequence of an application to $P_{n}$ of Theorem 2 .

THEOREM 4. The generating function $P(x)=\sum_{n=0}^{\infty} \phi\left(P_{n}\right) x^{n}$ for the polynomials $\phi\left(P_{n}\right)$ is $\left(1-\lambda x+x^{2}\right)^{-1}$.

Proof. Since $\phi\left(P_{1}\right)=\lambda$ and $\phi\left(P_{2}\right)=\lambda^{2}-1$ we can consistently define $\phi\left(P_{0}\right)=1$. Multiplying (8) by $x^{n}$ and summing from $n=2$ we have

$$
\sum_{n=2}^{\infty} \phi\left(P_{n}\right) x^{n}=\lambda x \sum_{n=2}^{\infty} \phi\left(P_{n-1}\right) x^{n-1}-x^{2} \sum_{n=2}^{\infty} \phi\left(P_{n-2}\right) .
$$


We can rewrite (9) as

$$
P(x)-x \phi\left(P_{1}\right)-\phi\left(P_{0}\right)=\lambda x\left\{P(x)-\phi\left(P_{0}\right)\right\}-x^{2} P(x),
$$

whence

$$
\left(1-\lambda x+x^{2}\right) P(x)=x \phi\left(P_{1}\right)+\phi\left(P_{0}\right)-\lambda x \phi\left(P_{0}\right)=1,
$$

which proves the theorem.

Finally we may note the following result concerning trees:

THEOREM 5. The characteristic polynomial of a tree having an odd (even) number of nodes contains only odd (even) powers of $\lambda$.

Proof. If $G$ has $n$ nodes, then $G-u$ has $n-1$ nodes, and $G-u-v$ has $n-2$ nodes. The theorem then follows readily by induction on application of Theorem 2 .

The example of Fig. 9 shows that the converse of Theorem 5 is not true, for $\phi(G)$ can be an odd or even function of $\lambda$ when $G$ is not a tree.

\section{Conclusion}

Although it is abundantly clear from the foregoing that the characteristic polynomial is not sufficient to distinguish between non-isomorphic graphs, it can be used effectively as a heuristic when perfect accuracy is not essential. Table 1 gives the number of graphs, connected graphs, cospectral pairs (including those arising from triads and cospectral connected pairs with $p \leqslant 7$ points, denoted by $N(p)$, $C(p), S_{0}(p), S_{1}(p)$, respectively.

TABLE 1

\begin{tabular}{lrrcccc}
$p$ & $N(p)$ & $C(p)$ & $S_{0}(p)$ & $S_{1}(p)$ & $f_{0}(p)$ & $f_{1}(p)$ \\
\hline 2 & 2 & 1 & 0 & 0 & 0 & 0 \\
3 & 4 & 2 & 0 & 0 & 0 & 0 \\
4 & 11 & 6 & 0 & 0 & 0 & 0 \\
5 & 34 & 21 & 1 & 0 & $0.18 \times 10^{-2}$ & 0 \\
6 & 156 & 112 & 5 & 1 & $0.41 \times 10^{-3}$ & $0.82 \times 10^{-4}$ \\
7 & 1044 & 853 & 58 & 33 & $0.11 \times 10^{-3}$ & $0.61 \times 10^{-4}$
\end{tabular}

The probability that a pair of graphs chosen at random from the collection of non-isomorphic ones with $p$ nodes are cospectral is given by $f_{0}(p) ; f_{1}(p)$ is defined analogously for connected graphs. One is tempted to conjecture that $f_{0}(p)$ and $f_{1}(p)$ are decreasing functions of $p$ for $p \geqslant 6$.

The situation is somewhat similar for trees. Letting $g(p)$ denote the probability that a pair of non-isomorphic $p$-node trees is cospectral, we find

$$
g(p)= \begin{cases}0 & \text { if } p \leqslant 7 \\ 0.40 \times 10^{-2} & \text { if } p=8 \\ 0.46 \times 10^{-2} & \text { if } p=9 \\ 0.72 \times 10^{-3} & \text { if } p=10\end{cases}
$$


Added in proof.

Collatz and Sinogowitz [1] found explicitly:

$$
\phi\left(P_{n}\right)=\sum_{m=0}^{[n / 2]}(-1)^{m}\left(\begin{array}{c}
n-m \\
m
\end{array}\right) \lambda^{n-2 m}
$$

which also follows from (8) or (9).

There are additional references [8-12] on the spectrum of a graph. Fisher [8] produced a pair of cospectral graphs with the same partition having 15 points and no endpoints or cutpoints, unlike Figure 7. Sachs [9] and Spialter [11] analyzed the coefficients of the characteristic polynomial in terms of the structure of the graph. Smith [10] gave a criterion for a graph to have just one positive eigenvalue. And Turner [12] proved that much stronger conjectures than our original one do not hold.

\section{References}

1. L. Collatz and U. Sinogowitz, "Spektren endlicher Graphen ”, Abh. Math. Sem. Univ. Hamburg, 21 (1957), 64-77.

2. F. Harary, Graph theory (Addison-Wesley, Reading, Mass., 1969).

3. - R. Norman and D. Cartwright, Structural models: An introduction to the theory of directed graphs (Wiley, New York, 1965).

4. C. King, Characteristic polynomials of 7-point graphs, Scientific Report UWI/CC 6, University of the West Indies, Kingston, 1967.

5. A. Mowshowitz, "The characteristic polynomial of a graph ", J. Combinatorial Theory, to appear.

6. R. C. Read, The production of a catalogue of digraphs on 5 nodes. Scientific Report UWI/CC 1, University of the West Indies, Kingston, 1966.

7. A. A. Zykov, " On certain properties of linear complexes ", Amer. Math. Soc. Translation No. 79 (1952). Translated from Mat. Sbornik N.S., 24 (66) (1949), 163-188.

8. M. Fisher, "On hearing the shape of a drum ", J. Combinatorial Theory, 1 (1966), 105-125.

9. H. Sachs, "Beziehungen zwischen den in einem Graphen enthaltenen Kreisen und seinem charakteristischen Polynom ", Publ. Math. Debrecen, 11 (1964), 119-134.

10. J. H. Smith, "Some properties of the spectrum of a graph ", Combinatorial structures and their applications (R. K. Guy, et al., eds.), Gordon and Breach, New York, 1970, 403-406.

11. L. Spialter, "The atom connectivity matrix and its characteristic polynomial ", J. Chem. Documentation, 4 (1964), 261-274; J. Amer. Chem. Soc., 85 (1963), 2012-2013.

12. J. Turner, "Generalized matrix functions and the graph isomorphism problem", SIAM J. Appl. Math., 16 (1968), 520-526.

University of Michigan.

University of the West Indies.

University of British Columbia.

University of Waterloo. 